\title{
A study of fine structure of diffuse aurora with ALIS-FAST measurements
}

\author{
T. Sergienko ${ }^{1}$, I. Sandahl ${ }^{1}$, B. Gustavsson ${ }^{2}$, L. Andersson ${ }^{3}$, U. Brändström ${ }^{1}$, and Å. Steen ${ }^{4}$ \\ ${ }^{1}$ Swedish Institute of Space Physics, Kiruna, Sweden \\ ${ }^{2}$ University of Troms $\varnothing$, Norway \\ ${ }^{3}$ University of Colorado, Boulder, USA \\ ${ }^{4}$ RemSpace group, Kvillsfors, Sweden
}

Received: 21 April 2008 - Revised: 15 August 2008 - Accepted: 27 August 2008 - Published: 21 October 2008

\begin{abstract}
We present results of an investigation of the fine structure of the night sector diffuse auroral zone, observed simultaneously with optical instruments (ALIS) from the ground and the FAST electron spectrometer from space 16 February 1997. Both the optical and particle data show that the diffuse auroral zone consisted of two regions. The equatorward part of the diffuse aurora was occupied by a pattern of regular, parallel auroral stripes. The auroral stripes were significantly brighter than the background luminosity, had widths of approximately $5 \mathrm{~km}$ and moved southward with a velocity of about $100 \mathrm{~m} / \mathrm{s}$. The second region, located between the region with auroral stripes and the discrete auroral arcs to the north, was filled with weak and almost homogeneous luminosity, against which short-lived auroral rays and small patches appeared chaotically. From analysis of the electron differential fluxes corresponding to the different regions of the diffuse aurora and based on existing theories of the scattering process we conclude the following: Strong pitch angle diffusion by electron cyclotron harmonic waves $(\mathrm{ECH})$ of plasma sheet electrons in the energy range from a few hundred $\mathrm{eV}$ to $3-4 \mathrm{keV}$ was responsible for the electron precipitation, that produced the background luminosity within the whole diffuse zone. The fine structure, represented by the auroral stripes, was created by precipitation of electrons above $3-4 \mathrm{keV}$ as a result of pitch angle diffusion into the loss cone by whistler mode waves. A so called "internal gravity wave" (Safargaleev and Maltsev, 1986) may explain the formation of the regular spatial pattern formed by the auroral stripes in the equatorward part of the diffuse auroral zone.
\end{abstract}

Keywords. Ionosphere (Particle precipitation; Waveparticle interactions) - Magnetospheric physics (Auroral phenomena)

Correspondence to: T. Sergienko

(tima.sergienko@irf.se)

\section{Introduction}

Diffuse aurora is the most common and the most widespread type of aurora. The optical definition of diffuse aurora was introduced by Lui and Anger (1973). The first space-borne imager, the one on ISIS-2, revealed it as a belt of fairly uniform luminosity of low intensity that was seen in basically every orbit. In the night sector the diffuse auroral zone is located equatorward of auroral oval of discrete forms and its width is controlled by substorm activity and by the interplanetary magnetic field $B_{z}$ component (Feldstein and Galperin, 1985). Precipitation of plasma sheet electrons is thought to be responsible for the diffuse aurora. Taking into account that the diffuse aurora occupies a wide area and exists almost permanently, this electron precipitation is a major source of loss of magnetospheric particles at $\sim \mathrm{keV}$ energies (Lyons et al., 1999).

The mechanisms causing the precipitation of the magnetospheric particles responsible for the diffuse aurora have been under debate for several decades. There are two main candidates. The first is that the particle precipitation is a result of pitch angle diffusion due to interaction of particles with electrostatic electron cyclotron harmonic $(\mathrm{ECH})$ waves (Kennel and Petscheck, 1966; Bespalov and Trakhtengerts, 1986; Johnstone et al., 1993; Villalón and Burke, 1995). The alternative theory is electron scattering by whistler mode waves (Kennel et al., 1970; Lyons, 1974; Horne and Thorne, 2000). The main argument against the first theory has been that the power of the ECH waves measured at geostationary orbit is not sufficient to cause strong pitch angle diffusion (Belmont et al., 1983). The main problem with the second mechanism was that, although Johnstone et al. (1993) showed that whistler mode waves can resonate with $\mathrm{keV}$ electrons in the frequency band below the electron gyro frequency, relatively high temperature anisotropies are required to generate such waves. 


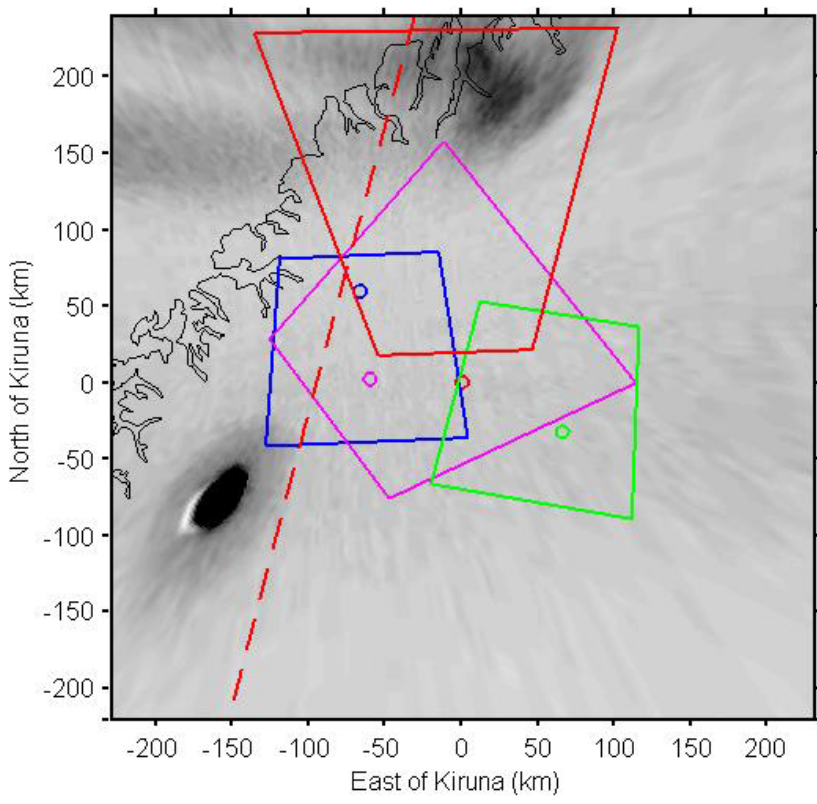

Fig. 1. Geography of the experiment. The coordinate system is a cartesian system centred in Kiruna. The $\mathrm{X}$-axis is directed to geographic East, the Y-axis to geographic North. The background is the FMI camera image projected to $110 \mathrm{~km}$ (Negative). Colour circles and frames indicate location and field of view of ALIS cameras projected to $110 \mathrm{~km}$. Red - Kiruna, magenta - Nikkaluokta, blue - Abisko, green - Silkkimuotka. The dashed red line is the FAST magnetic footprint at $110 \mathrm{~km}$. The black spot in the lower left section of the figure is the moon.

Recently, using data from the wave and particle experiments on the CRRES satellite, Meredith et al. (2000) studied the evolution of electron pitch angle distributions in the equatorial plane and their relation to $\mathrm{ECH}$ and whistler mode wave activity. They could show that both types of waves are responsible for the electron precipitation causing the diffuse aurora. This result was supported by numerical calculations conducted by Horne et al. (2003) for analysis of whistler mode hiss, chorus, and ECH waves during a substorm injection event. The authors found that ECH waves interact strongly with electrons in the energy range from few hundred $\mathrm{eV}$ to a few $\mathrm{keV}$, while whistler mode hiss effectively scatters electrons with energy higher than $\sim 3 \mathrm{keV}$, and chorus interacts with particle with energy less than a few hundred $\mathrm{eV}$.

For a long time many satellite and ground-based measurements gave a description that the diffuse aurora is spatially uniform. However, modern sensitive high resolution optical measurements show that diffuse electron aurora can contain a fair amount of fine structure. Studies regarding this are often published under the heading black aurora. The term black aurora is used for relatively small, well defined regions with a distinct reduction of luminosity within large-scale regions of otherwise homogeneous, diffuse aurora (Røyrvik, 1976;
Davis, 1978). Peticolas et al. (2002) studied black aurora using FAST plasma and field measurements and optical TV observations and found that black aurora occurred in narrow spatial regions where electrons had partial double loss cones, while the electrons in the ambient broad region of diffuse aurora were characterized by single loss cones. They suggested that this signature of black aurora can be explained by pitch angle diffusion of electrons in the diffuse aurora due to interaction with both ECH and whistler mode waves, and suppression of the whistler mode waves in the region of black aurora. They also concluded, that in the central plasma sheet region of the pre-midnight auroral oval, partial double loss cones are common, implying that fine structure of the diffuse aurora can be explained by spatial (and temporal) modulation of the efficiency of whistler mode wave-particle interaction.

In our paper we investigate the fine structure of the diffuse aurora in evening sector using conjugate high resolution optical and particle data.

\section{Instrumentation}

This study is based on data from ALIS (Auroral Large Imaging System), an FMI (Finnish Meteorological Institute) digital all sky camera, and the FAST satellite, obtained 16 February 1997 when FAST passed over ALIS.

ALIS consists of unmanned imaging stations located in Northern Scandinavia in a grid of about $50 \times 50 \mathrm{~km}$. Each station is equipped with an imager having a high-resolution monochrome $1024 \times 1024$ pixel CCD detector and a filter wheel with six positions for narrow-band interference filters. The field of view is 70 degrees diagonally for most imagers, but there are also two units with a 90 degrees field of view. The imagers are mounted in a positioning system and can be pointed so that several imagers can view a common volume. Detailed information about ALIS is given by Brändström (2003) and is also found at http://alis.irf.se/.

In the present experiment data from three ALIS stations were used; Abisko ( $\left.68.36^{\circ} \mathrm{N}, 18.82^{\circ} \mathrm{E}\right)$, Silkkimuotka $\left(68.03^{\circ} \mathrm{N}, 21.69^{\circ} \mathrm{E}\right)$ and Nikkaluokta $\left(67.85^{\circ} \mathrm{N}, 19.00^{\circ} \mathrm{E}\right)$. The image size for the mode used was $512 \times 512$ pixels, giving a spatial resolution of approximately $200 \mathrm{~m}$ at $100 \mathrm{~km}$ for the 70 degree cameras, and $300 \mathrm{~m}$ for the 90 degrees cameras. All images were obtained using a $557.7 \mathrm{~nm}$ filter with a bandwidth of $4 \mathrm{~nm}$, the temporal resolution was $30 \mathrm{~s}$, and the expose time was $1 \mathrm{~s}$. Figure 1 shows the location of the ALIS stations and the fields of view at $110 \mathrm{~km}$ altitude.

For a general overview of the behaviour of the aurora we use data from the FMI all sky camera located in Abisko. During the experiment the FMI all-sky camera operated with a $557.7 \mathrm{~nm}$ filter taking one image every $20 \mathrm{~s}$ with exposure time of $520 \mathrm{~ms}$. A detailed description of this camera is presented at http://www.ava.fmi.fi/MIRACLE/ASC/ and by Syrjäsuo (2001). 


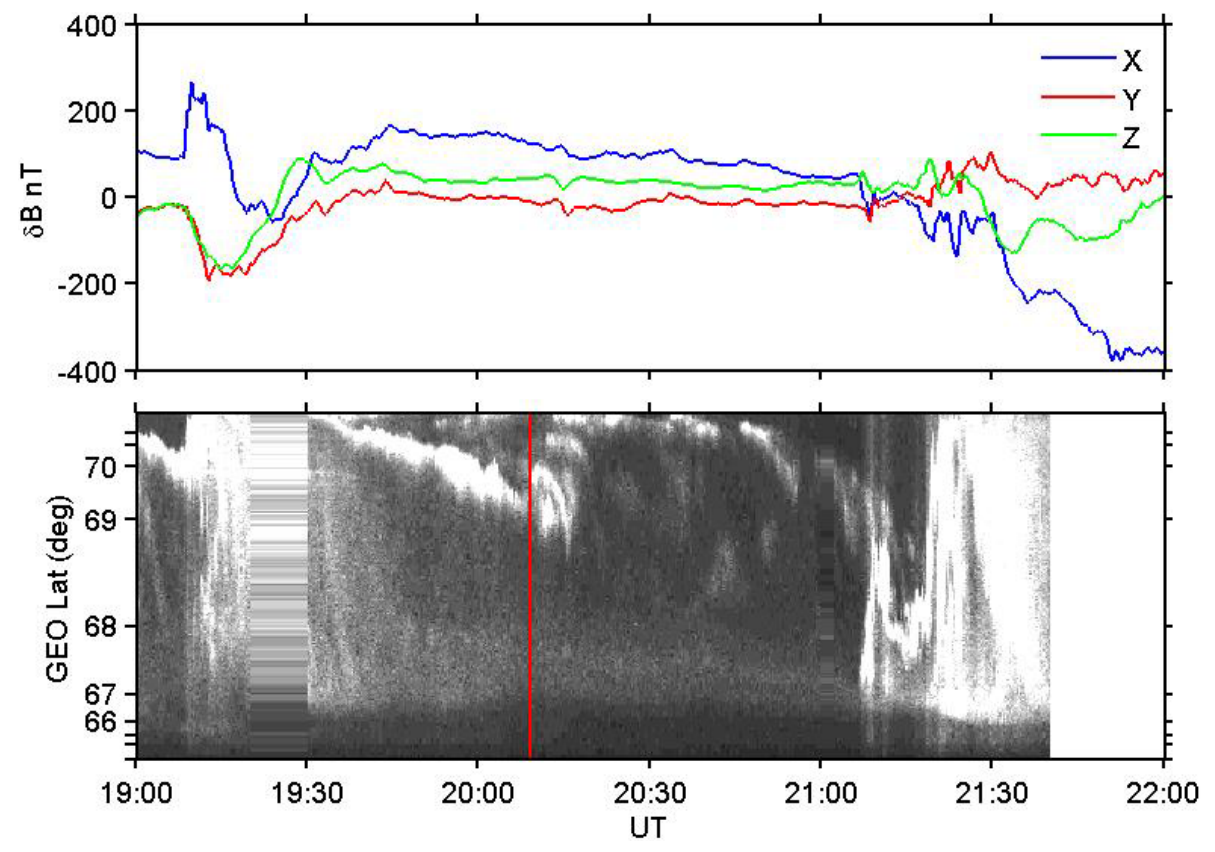

Fig. 2. Upper panel - variations of the magnetic field obtained from Kiruna magnetometer data. Bottom panel - keogram from images obtained by the FMI camera in Abisko at the $557.7 \mathrm{~nm}$ emission. The vertical red line indicates the time of the FAST pass.

Electron and ion data were obtained by electrostatic analyzers onboard the FAST satellite (Carlson et al., 1998). During this event the particle fluxes were sampled at 48 energy levels $(4 \mathrm{eV}$ to $32 \mathrm{keV})$ and 32 pitch angles $\left(0-360^{\circ}\right)$ every $0.32 \mathrm{~s}$. Taking into account the FAST altitude of $3400 \mathrm{~km}$, the spatial resolution of the particle data at $100 \mathrm{~km}$ altitude is approximately $400 \mathrm{~m}$, that is close to the spatial resolution given by the ALIS cameras.

\section{Data overview}

\subsection{Optical observations}

Figure 2 shows data from the Kiruna magnetometer and a keogram from FMI camera images at $557.7 \mathrm{~nm}$. The keogram was obtained along the projection of the FAST footprint at an altitude of $110 \mathrm{~km}$ onto the FMI camera images. The FAST track is shown by a red line in Fig. 3 together with the all-sky image obtained at 20:09:20 UT. The time of the FAST pass is shown by a red vertical line in Fig. 2. From the keogram and the image of the aurora it is seen that FAST crossed three clearly distinguishable zones of auroral luminosity. The most equatorward region is a low intensity diffuse auroral band. The northern part of the auroral image is occupied by discrete auroral arcs. In the FMI camera image the region between the diffuse band and the auroral arcs appears to be free of any auroral forms, but, as it will be shown by the data of ALIS with its much higher sensitivity, this re- gion is filled by weak auroral forms such as patches, rays, and arc fragments.

FAST moved from south to north and passed the field of view of the ground-based imagers around 20:10 UT. Approximately one hour before and one hour after this moment substorm activations were observed in both magnetometer and optical measurements. From about 19:30 UT the keogram shows equatorward moving discrete auroral arcs that formed during the recovery phase of the first substorm. A few minutes after the FAST passage these discrete auroral arcs moved quickly northward and then again moved equatorward until the next substorm breakup. The behaviour of the diffuse aurora was more stable. The wide auroral band narrowed with time while the center position of the band did not change significantly between the two substorms. Such behaviour of the auroral pattern is typical for the substorm growth phase in the evening sector. This equatorward diffuse auroral zone in the pre-midnight sector is the topic of our study.

Figure 4 presents three consecutive images of aurora obtained by ALIS when the FAST footprint was inside the field of view of the instruments. Each plot is composed of images projected to the same altitude from the three ALIS cameras located in Abisko, Nikkaluokta and Silkkimuotka. We here only consider the images from the three stations that observe the diffuse auroral zone, though, as it is seen from Fig. 1, the Kiruna camera, that was directed northward, provided images of the discrete auroral arcs also.

As the ALIS camera fields of view overlap it is possible to estimate the height of the auroral forms by fitting 


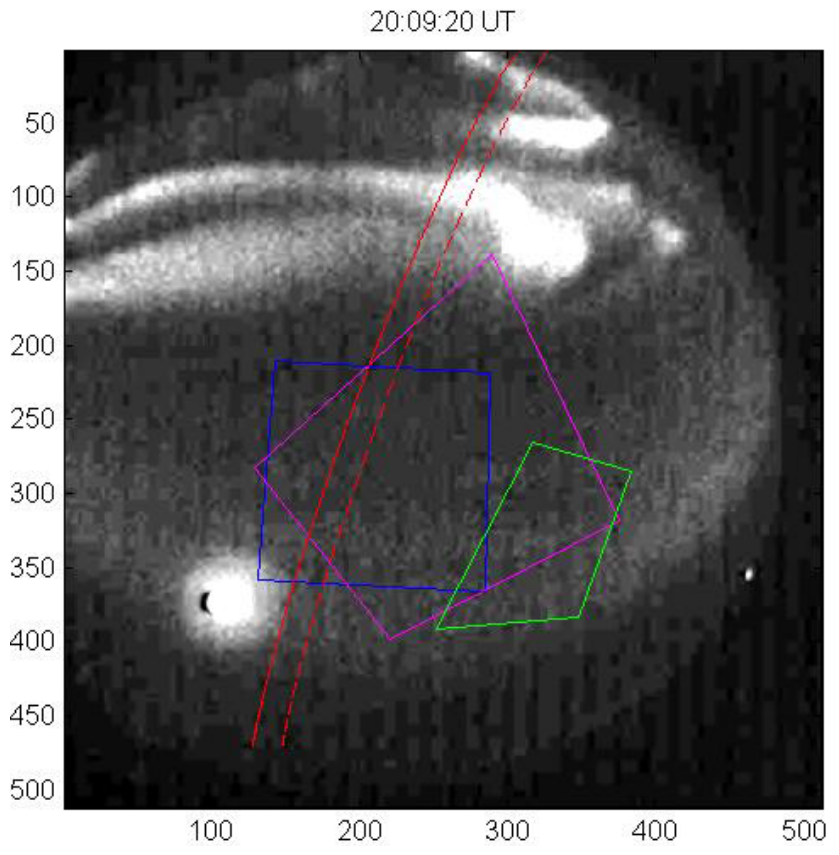

Fig. 3. The FMI camera image at 20:09:20 UT. Colour frames show projections of ALIS camera fields of view at $110 \mathrm{~km}$. Blue - Abisko, magenta - Nikkaluokta, green - Silkkimuotka. The solid red line shows the FAST footprint. The dashed red line indicates the line along which the keogram in Fig. 2 was obtained. This shifting was done to avoid the influence of the moon (the bright spot in lower left corner).

the projection altitude to reach the best matching of auroral images taken from different positions. For the auroral stripes the best result was obtained for a projection altitude of $107 \mathrm{~km}$. This is the altitude, that was used for plotting the images in Fig. 4.

In the images obtained by ALIS the diffuse auroral band, that appears to be homogeneous in the all sky images, has clearly recognizable fine structure. This auroral region consists of thin auroral stripes forming a very regular spatial structure. The stripes stretch along the geomagnetic latitude, have a width of about $5 \mathrm{~km}$ and are separated by stripes with only background luminosity of approximately the same width. The intensity of each stripe does not vary significantly in longitude but changes with time. Since the ALIS cameras took one image every $30 \mathrm{~s}$ during this event we can not say anything about short time intensity variations with periods of $1-60 \mathrm{~s}$ that are typical for auroral pulsations. However, the system of auroral stripes as a whole structure varied in intensity with period of $\sim 5-6 \mathrm{~min}$. The auroral stripes moved gradually equatorward with a velocity of approximately of $100 \mathrm{~m} / \mathrm{s}$. The stripe pattern remained quite stable during about $20 \mathrm{~min}$.

The region to the north of the auroral stripes, that looks empty of aurora in the all sky image, is, in reality, filled by a mixture of different auroral forms. These forms have very low intensities and look like short-lived auroral rays and small patches appearing chaotically against the weak diffuse background.

\subsection{Particle data}

The auroral particle data measured by instruments on the FAST satellite on 16 February 1997 (orbit 1940) are presented in Fig. 5. The panels from top to bottom represent energy spectra of the downward, trapped, and upward electrons. The ion data are not considered because the energy fluxes of precipitating ions did not exceed $20 \%$ of the electron energy flux and did not demonstrate any fine structure in the interval of interest. The downward electron spectra were calculated by integration of the measured differential energy fluxes within the atmospheric loss cone. The upward and trapped electron spectra were obtained by integration within the upward loss cone, and outside the loss cones, respectively. The loss cone was determined from the measured pitch angle distributions and is equal to 33 degrees.

Similar to the optical data, the electron spectrograms can be divided into three well-defined regions with clear borders between them. The most poleward region, after 20:10:14 UT, consists of multiple inverted- $\mathrm{V}$ structures formed by accelerated electrons with peak energy of $1-4 \mathrm{keV}$. These electrons are responsible for formation of the multiple bright auroral arcs that occupy the northward part of the all-sky images.

The electron energy fluxes in the regions south of the inverted-V structures had much lower intensities. In order to show the details, this part of the spectrogram is plotted separately in Fig. 6. It is clearly seen that there are two populations with different characteristics in the differential energy fluxes. The two populations overlap between 20:09:08 UT and 20:09:37 UT. The boundary at 20:09:37 UT corresponds to the boundary 2e defined by Newell et al. (1996) Boundary $2 \mathrm{e}$ is where $d E_{e} / d \lambda$ ( $E_{e}$ - the average energy of electrons, $\lambda$ - the latitude) changes sign from plus to minus. The more poleward, rather featureless, population belongs to the main (central) plasma sheet. Its average energy decreases slowly with decreasing latitude in agreement with the well known fact that low energy electrons in the plasma sheet reach closer to Earth than do the high energy electrons. The other electron population, before 20:09:37 UT, has higher energies. The plasma sheet electrons do not demonstrate significant variations in neither downward nor trapped differential energy fluxes. In contrast, the energy fluxes of the high energy electron population varied strongly. The variations appear mainly in the downward energy fluxes where they are very pronounced and have a periodic structure. Comparing the particle and the optical data we can conclude that this structured electron precipitation was responsible for the auroral stripes, while the electrons from the central plasma sheet were connected with the low intensity diffuse aurora 


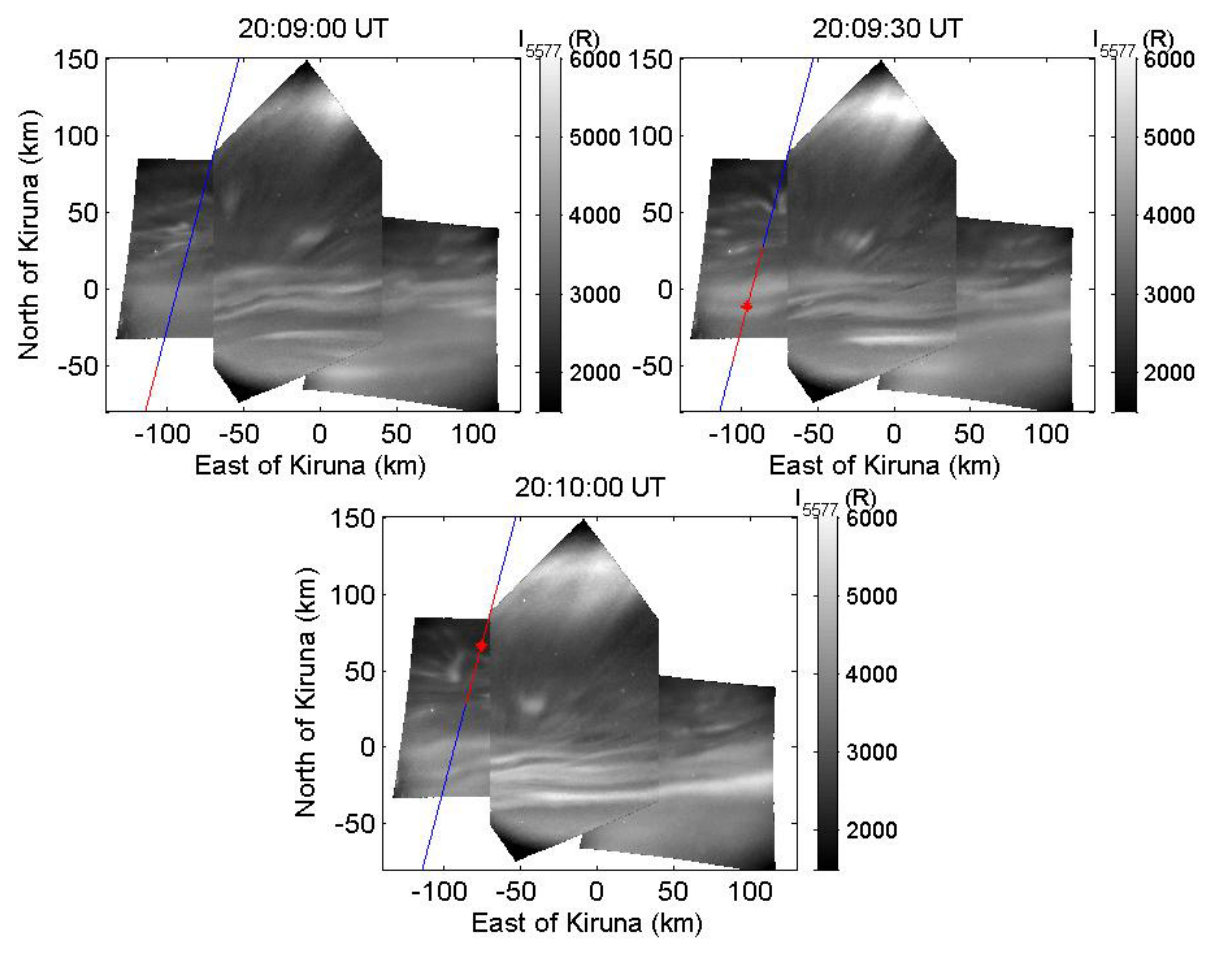

Fig. 4. Abisko, Nikkaluokta, and Silkkimuotka ALIS images of the aurora projected to $107 \mathrm{~km}$. The blue lines show the FAST footprint. Red asterisks indicate the position of FAST at the moment when the image was taken. Red lines indicate the time interval $\pm 15 \mathrm{~s}$ around this time.

between the stripe zone and the zone with discrete auroral arcs.

\section{Discussion}

Comparison of the optical and electron data allows us to suggest that the auroral stripe structure in the equatorward part of the auroral images was created by high energy auroral electrons with ordered structure in the downgoing electron spectra. To prove this suggestion we calculated altitude profiles of the $557.7 \mathrm{~nm}$ emission along the FAST track. In the calculation two models were used, a Monte-Carlo simulation of the auroral electron transport (Sergienko and Ivanov, 1993) and a model of the auroral green line (Ivanov et al., 1993). The initial electron fluxes for the electron transport code were obtained from the FAST data by mapping the downward fluxes from the FAST altitude down to $500 \mathrm{~km}$ taking into account the Earth's magnetic field convergence. The MSIS90 model (Hedin, 1991) was used to describe the neutral atmosphere in both models. The results of the calculations are presented in Fig. 7. The top panel shows the differential electron flux of downward electrons at $500 \mathrm{~km}$, the middle panel the calculated volume emission rate, and the bottom panel the calculated column intensity and optical measurements by ALIS. Good agreement between the measured optical data and the calculated luminosity in both positions and absolute intensities are demonstrated. The altitude of the maximum of the calculated $557.7 \mathrm{~nm}$ intensity inside the auroral stripes is about $107 \mathrm{~km}$ in excellent agreement with the height obtained from the optical data. From the calculated emission intensity it is also seen that the plasma sheet electrons create homogenous luminosity in the whole diffuse zone, while the discrete auroral features are created by fluctuations of the high energy part of the downward electron spectra.

We will now examine the pitch angle distributions inside and outside the auroral stripes and in the diffuse zone without stripes. We choose the stripe that was crossed by FAST at 20:09:29 UT and the minimum at 20:09:27 UT. Figure 8 shows differential energy fluxes as a function of energy and pitch angle (top) and the energy flux as a function of energy for downward and trapped particles (bottom). The columns from left to right correspond to fluxes between stripes (20:09:27 UT), in the auroral stripe (20:09:29 UT), and in the region of lower intensity diffuse aurora (20:09:43 UT). The electron distributions with energy less than $4 \mathrm{keV}$ are isotropic in all three spectrograms for pitch angles less than 150 degrees and have a single loss cone (upward). In contrast, the pitch angle distributions of electrons with energy more than $4 \mathrm{keV}$ have different character in different regions. The high energy fluxes outside the stripe, as well as in the low intensity diffuse aurora, have double loss cones, while 


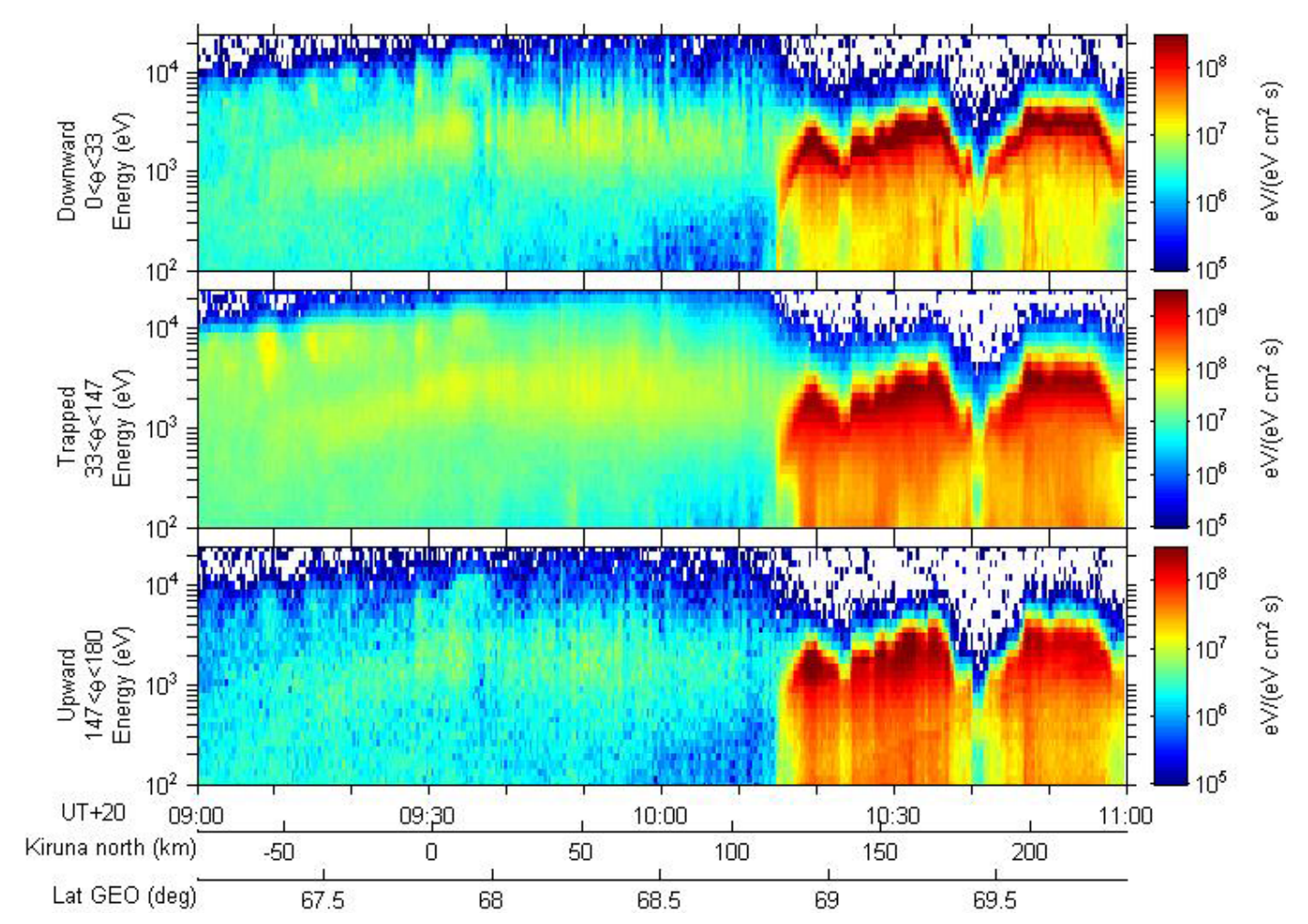

Fig. 5. The electron differential energy flux as a function of time and energy for the time interval 20:09:00-20:11:00 UT. The upper panel presents the fluxes integrated over the pitch angle interval $0<\theta<33$ (downward), the middle panel the interval $33<\theta<147$ (trapped), and the bottom panel the interval $147<\theta<180$ (upward).

inside the stripe the downward loss cone is partly filled and the fluxes inside the loss cone are anisotropic.

For quantitative comparison, the electron spectra presented in the bottom panel of Fig. 8 were normalized to unit solid angle. Inside the auroral stripes there are no differences between the downgoing and the trapped electron spectra at any energy. Outside the stripes differences between the spectra appear at the energy where the precipitating loss cone becomes partly filled. This indicates that electrons were not acted upon by any acceleration mechanism and, most probably, that the electron precipitation was caused by pitch angle diffusion. The fact that filling of the loss cone depends on the electron energy and also has a spatial variation shows that this mechanism is energy sensitive and that its efficiency varies in space.

In order to investigate the variations of the pitch angle diffusion at different energies and locations we calculated the anisotropy coefficient, that we define as the ratio of trapped to downward electron fluxes, at every energy and time. By this definition, an anisotropy coefficient equal to one indicates that the precipitating loss cone is completely filled and corresponds to strong pitch angle diffusion. Values of the coefficient greater than one correspond to a partly filled or empty loss cone (weak diffusion), and values less than one indicate downward field-aligned acceleration. The anisotropy coefficient as a function of time (or distance along the FAST foot print) and energy is presented in Fig. 9 (bottom panel) together with spectra of the downward (top panel) and trapped (middle panel) electrons. It is clearly seen that the anisotropy coefficients are close to one at energies less than $3-4 \mathrm{keV}$ everywhere except the $3 \mathrm{~s}$ interval around 20:09:35 UT. At higher energies the anisotropy coefficient grows with energy but this increase has very different characters in different regions. To demonstrate this difference more clearly, the anisotropy coefficients for selected times (distances) are plotted versus energy in Fig. 10. The left diagram shows the anisotropy coefficients inside the auroral stripes (colour lines), and the right diagram corresponds to spectra between the stripes. The black lines give anisotropy coefficients obtained in the weak aurora region (at 20:09:49 UT). It is seen that the energy dependence of the anisotropies in the weak aurora region and in the interval between the auroral stripes are very similar. Inside the stripes, on the other hand, the anisotropy coefficients increase significantly more slowly or do not grow at all. To explain this difference we suggest that two mechanisms are responsible for the pitch angle diffusion. One of them acts effectively on the electrons with energy less than $\sim 3-4 \mathrm{keV}$ and works in the whole region. The other 


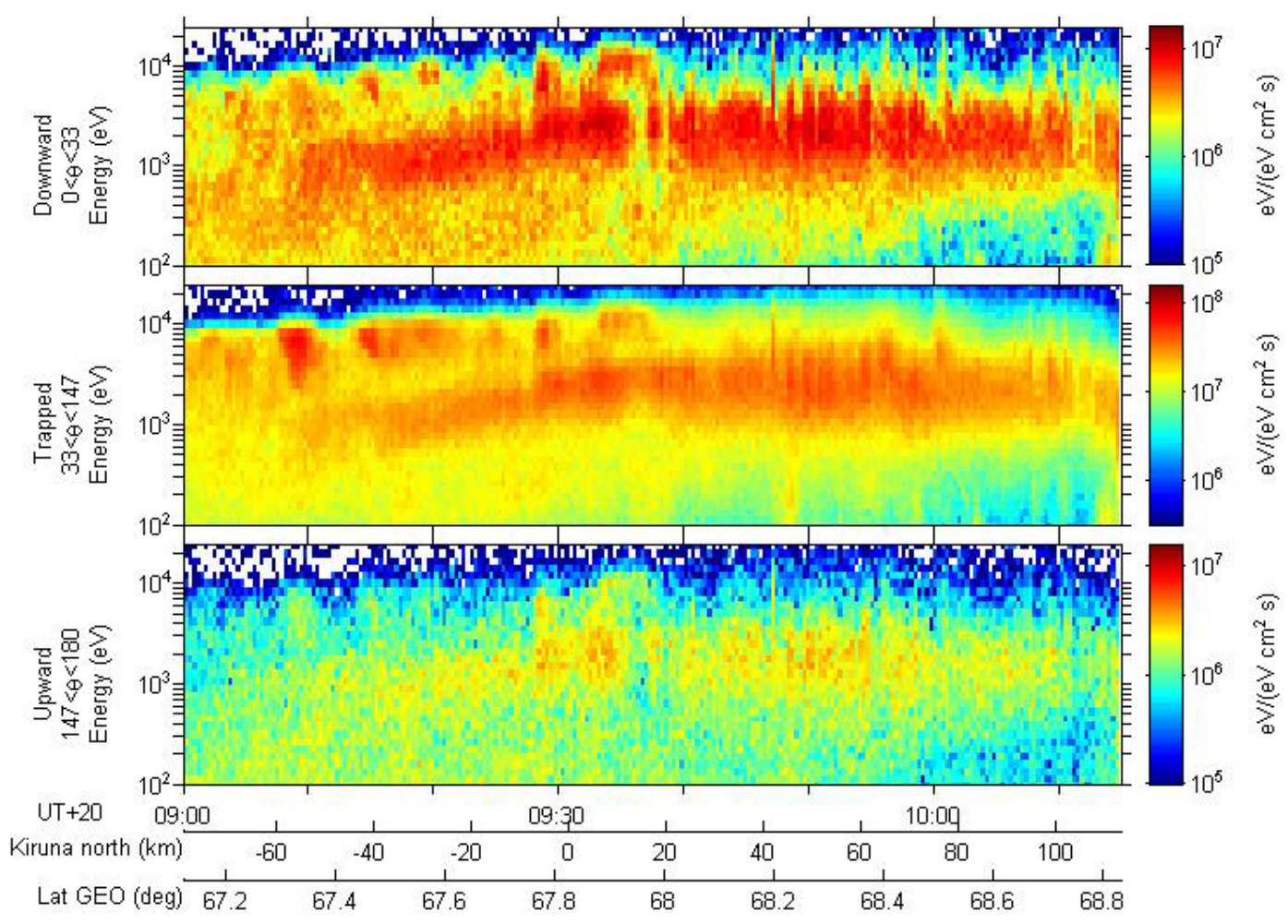

Fig. 6. Same as Fig. 5 but for the time interval 20:09:00-20:10:27 UT and with a different energy flux scale.

causes electron pitch angle diffusion at higher energies and its efficiency is modulated in space.

As described in the introduction, there are two mechanisms that are discussed in the literature as main pretenders for the pitch angle diffusion of plasma sheet electrons caused by wave-particle interactions. These are electron interaction with electromagnetic whistler mode waves (Kennel and Petscheck, 1966; Bespalov and Trakhtengerts, 1986; Johnstone et al., 1993; Villalón and Burke, 1995) and interaction with electrostatic electron cyclotron harmonic $(\mathrm{ECH})$ waves (Kennel et al., 1970; Lyons, 1974; Horne and Thorne, 2000). While the interaction of whistler mode waves with high energy electrons $(E>10 \mathrm{keV})$ was proved theoretically and experimentally, it was not clear that these waves would effectively interact with electrons at energies less than $10 \mathrm{keV}$ that are typical of the diffuse auroral zone. From the other side, the amplitude of ECH waves, measured at geostationary orbit, was concluded to be too small to cause strong pitch angle diffusion and hence diffuse auroral precipitation. However, more recently, it was shown experimentally (Meredith et al., 2000) and theoretically (Horne et al., 2003) that both types of waves are important in pitch angle diffusion of the plasma sheet electrons during the auroral activity.

From results of numerical modelling, Horne et al. (2003) showed that whistler mode waves (the hiss band of the whistler mode) interact effectively with electrons at energies typically higher than $3 \mathrm{keV}$, and that they do not resonate with electrons in the energy range $0.2-3 \mathrm{keV}$. They also showed that ECH waves strongly scatter electrons in the energy range from $0.1 \mathrm{keV}$ to a few $\mathrm{keV}$. The delimiting energy of $3 \mathrm{keV}$ that divides the electrons into two ranges, one for each of the above wave types, is in excellent agreement with the cutoff energy obtained in our experiment, namely the energy at which the anisotropy coefficient grows above 1. Therefore, we can suggest that the electron precipitation responsible for the homogenous low intensity auroral luminosity was caused by the interaction of plasma sheet electrons with $\mathrm{ECH}$ waves. We can also suggest that the auroral stripes forming the ordered structure were due to scattering of higher energy electrons by whistler mode waves.

The spatial structure of the diffuse aurora observed in our experiment is very similar to the detached auroral arcs observed by the ISIS- 2 satellite (Anger et al., 1978). From analysis of the auroral scanning photometer data on ISIS-2 it was found that the detached auroral arcs occur predominantly in the evening sector at times of recovery from moderate geomagnetic activity. The latitudinal width of the detached arc zone varies from $\sim 70$ to $200 \mathrm{~km}$. From data of precipitating particles (Wallis et al., 1979) and conjugate measurements of the ionospheric parameters by the Chatanika incoherent scatter radar (Vondrak et al., 1983) it has been shown that the precipitating electrons in the detached arcs have peak energy 


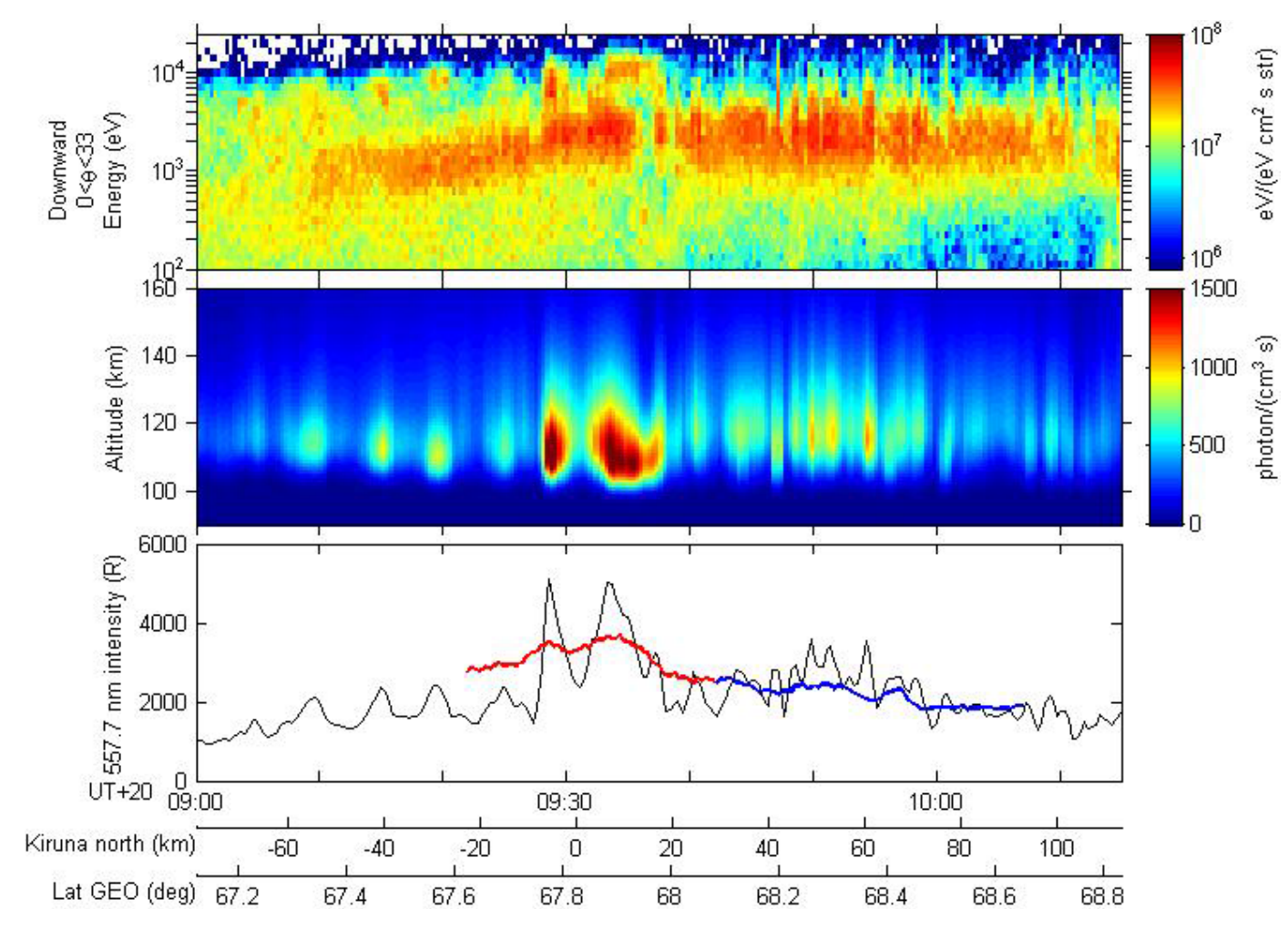

Fig. 7. Upper panel: Downward electron differential flux as a function of time and energy mapped down to $500 \mathrm{~km}$. Middle panel: Calculated altitude profiles of the volume emission rate of the $557.7 \mathrm{~nm}$ emission. Bottom panel: Column intensities of the $557.7 \mathrm{~nm}$ emission. The black line gives the calculated results. The red line shows intensities taken from the Abisko image at 20:09:30 UT. The blue line shows intensities taken from Abisko image at 20:10:00 UT.

near $6 \mathrm{keV}$, while the typical energies of the electrons in the nearby diffuse aurora are generally significantly lower. Considerable small scale spatial variations (units of $\mathrm{km}$ ) of the electron fluxes are usually present within the detached arc zone.

As a possible mechanism of the detached auroral arcs, Wallis et al. (1979) have proposed that trapped high energy electrons, residuary after a substorm injection at some earlier time, provide the source population for detached arc events. Pitch angle diffusion of these residual particles due to interaction with whistler mode waves was suggested to be the precipitation mechanism. This scenario is in very good agreement with our observations. It is clearly seen (Fig. 4) that inside the region of the auroral stripes the electrons belong to two different populations, with energy in the range of 1$2 \mathrm{keV}$ and $5-9 \mathrm{keV}$, respectively. The low energy population is a direct extension of the plasma sheet electrons, while the high energy particles are electrons residuary after the substorm that occurred approximately one hour before the moment of observations.

One more phenomenon closely related to diffuse aurora is the "black aurora". The term black aurora is used for relatively small, well defined regions with a distinct reduction of luminosity within large-scale regions of otherwise homogeneous, diffuse aurora (Røyrvik, 1976; Davis, 1978). In a paper by Peticolas et al. (2002) a case study of black aurora based on conjugate aircraft-based optical observations and plasma measurements by the FAST satellite was presented. The authors found that in the diffuse aurora, surrounding the black arc, the downward differential electron fluxes were isotropic at all energies creating a single loss cone distribution. In the region associated with the black aurora the differential electron fluxes were depleted in the downward loss cone at energies greater than $2 \mathrm{keV}$. It was suggested that in diffuse aurora both ECH waves and whistler mode, upper band chorus waves, were responsible for the electron precipitation, while the black aurora was a signature of depletion of the downward loss cone at high energies caused by suppression of the chorus waves.

An intriguing aspect of our observations is the very regular periodic spacing in latitude of the stripes. If the auroral stripes result from pitch angle diffusion of electrons by the hiss band of whistler mode waves, the spacing implies that there is modulation of either efficiency of wave excitation or efficiency of the pitch angle diffusion in the equatorial plane of the magnetosphere. Mapping the characteristic 

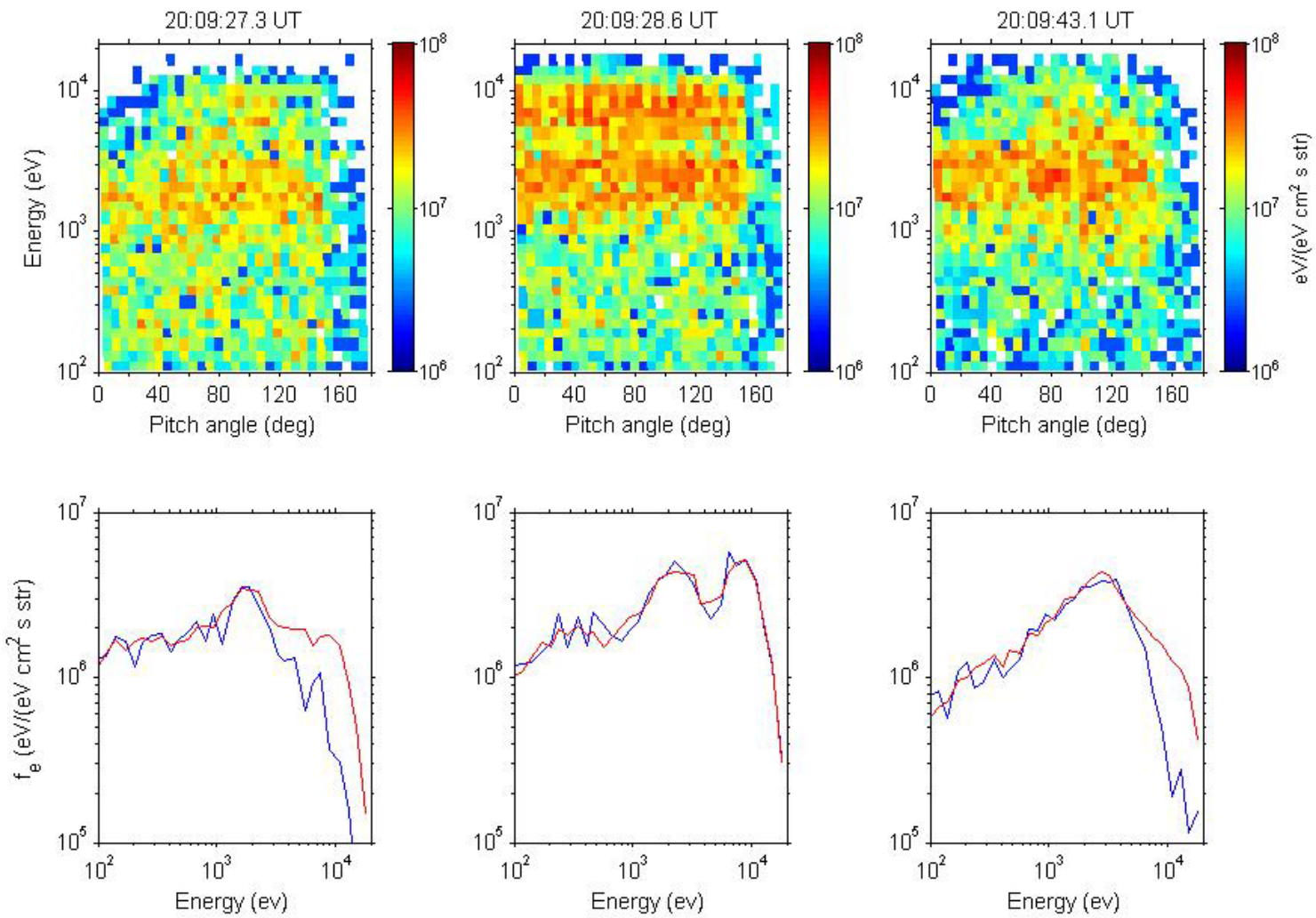

Fig. 8. Top panel: Differential electron energy fluxes as a function of pitch angle and energy. Bottom panel: Downward (blue) and trapped (red) electron energy fluxes as a function of energy. The left column represents a particle distribution between auroral stripes, the middle column a distribution inside a stripe, and the right column a distribution in the region of weak aurora.

scale of the auroral stripes $(\sim 10 \mathrm{~km})$ and their drift velocity $(\sim 100 \mathrm{~m} / \mathrm{s})$ to the equatorial plane gives approximately $5000 \mathrm{~km}$ scale and velocity of $50 \mathrm{~km} / \mathrm{s}$ in the magnetosphere. According to Horne et al. (2003) the hiss must be driven unstable by the temperature anisotropy of electrons at high energy, and, therefore, a compression magnetosonic wave can be a possible candidate to produce the periodic variation of the anisotropy. But for the present event this explanation seems less likely, since the typical Alfvén velocity in the equatorial plane for the $\mathrm{L}$ shell corresponding to the observation point is at least 10 times higher $(>500 \mathrm{~km} / \mathrm{s})$ than the mapped drift velocity. A proposed wave type with low enough velocity in the plasma sheet is the so called "internal gravity wave" that was described theoretically in a paper by Safargaleev and Maltsev (1986) in order to explain the propagation of bays in auroral riometer absorption. The authors showed that these waves propagate across the magnetic field with velocities of tens of $\mathrm{km} / \mathrm{s}$, in agreement with the value obtained from our observations.

\section{Conclusions}

We have presented results of an investigation of the fine structure of the pre midnight sector diffuse auroral zone observed simultaneously with sensitive imaging instruments from the ground (ALIS) and the FAST electron spectrometer from space on 16 February 1997.

Both the optical and particle data show that the diffuse auroral zone consisted of two regions. Most equatorward was a region filled with very regular parallel auroral stripes that extended along the magnetic latitude. The stripes were significantly brighter than the background luminosity, had a width of approximately $5 \mathrm{~km}$ and moved southward with a velocity of about $100 \mathrm{~m} / \mathrm{s}$. Between the region with the stripes and the region of discrete auroral arcs to the north, there was a region filled with weak and almost homogeneous luminosity, against which short-lived auroral rays and small patches appeared chaotically.

From analysis of the electron differential fluxes corresponding to the two different regions of the diffuse aurora we draw the following conclusions:

Strong pitch angle diffusion of the plasma sheet electrons in energy range from a few hundred $\mathrm{eV}$ to $3-4 \mathrm{keV}$ by $\mathrm{ECH}$ 


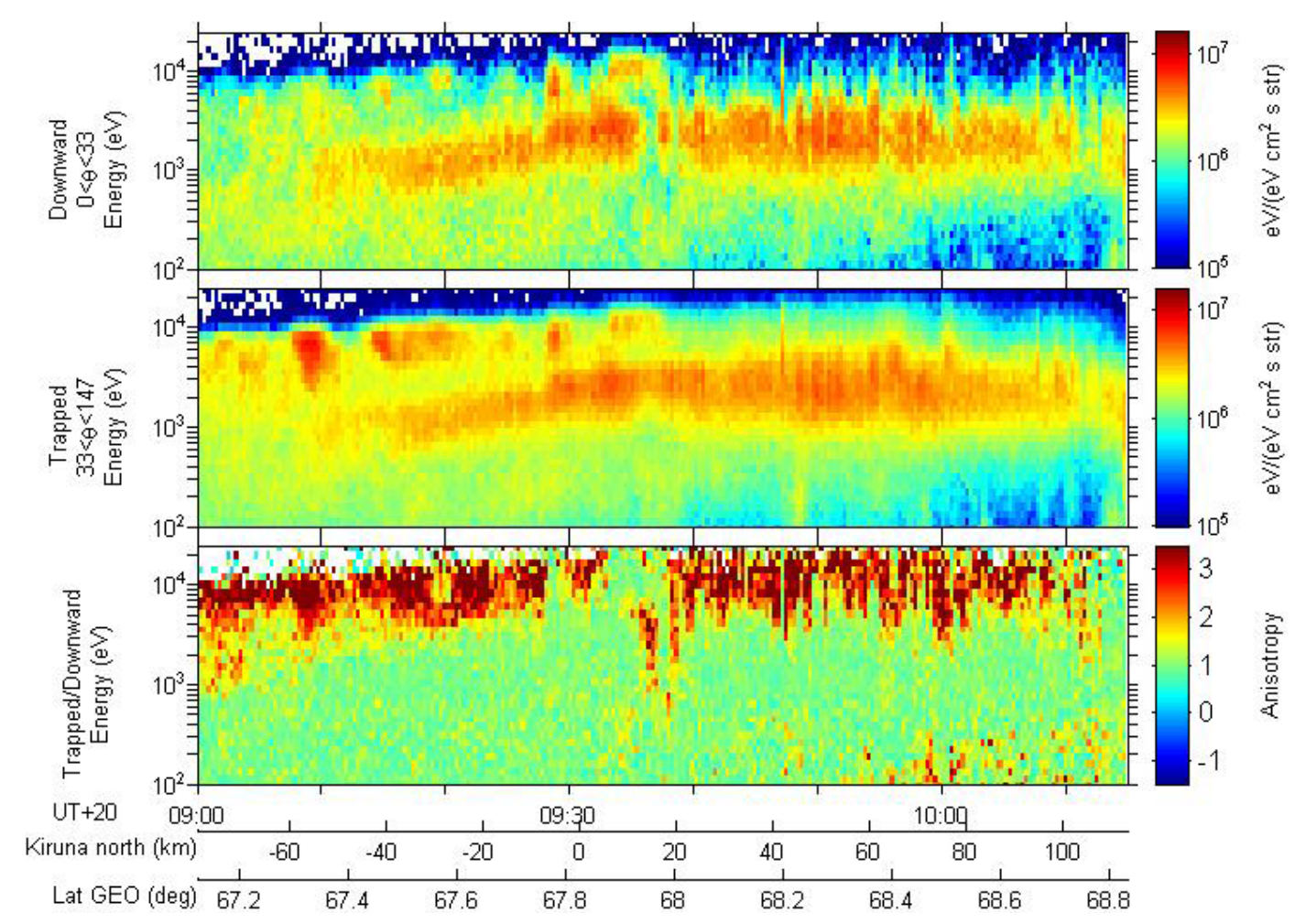

Fig. 9. Downward (upper panel) and trapped (middle panel) differential electron energy fluxes. The bottom panel shows anisotropy coefficients as function of energy and time. The anisotropy values can only be positive, but the pseudo colour scale varies from negative to positive values in order to contrast anisotropy variations at higher energies.
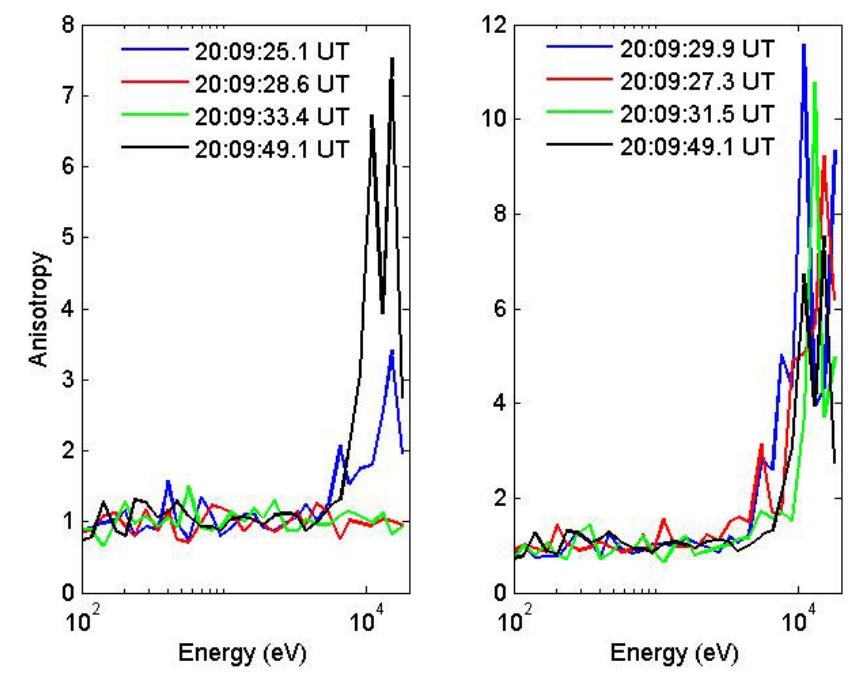

Fig. 10. The anisotropy coefficients as a function of energy. The left plot corresponds to regions inside the auroral stripes, the right plot corresponds to regions between the stripes. In both plots the black line shows the anisotropy coefficients in the region of weak aurora. waves was responsible for the electron precipitation that produced the background luminosity in the whole diffuse zone.

The more intense fine scale auroral forms were created by higher energy electrons precipitating as a result of pitch angle diffusion into the loss cone by whistler mode waves.

A so called "internal gravity wave", introduced by Safargaleev and Maltsev (1986) may explain the formation of the regular spatial structure formed by the auroral stripes in the equatorward part of diffuse auroral zone.

Acknowledgements. We gratefully acknowledge support for this research from ELSTATIK Foundation, Günter and Sylvia Lüttgens, and from the Swedish Research Council. We thank K. Kauristie (FMI) for providing ABK FMI ASC images for selected intervals. The FAST particle data were provided by SPRG, University of California http://sprg.ssl.berkeley.edu/fast/ (PI C. W. Carlson).

Topical Editor M. Pinnock thanks M. J. Kosch and K. Kaila for their help in evaluating this paper.

\section{References}

Anger, C. D., Moshupi, M. C., Wallis, D. D., Murphree, J. S., Brace, L. H., and Shepherd, G. G.: Detached auroral arcs in the trough region, J. Geophys. Res., 83, 2683-2689, 1978. 
Belmont, G., Fontaine, D., and Canu, P.: Are equatorial electron cyclotron waves responsible for diffuse auroral electron precipitation?, J. Geophys. Res., 88, 9163-9170, 1983.

Bespalov, P. A. and Trakhtengerts, V. Y.: Cyclotron Instability of the Earth Radiation Belts., Rev. Plasma Phys., 10, p. 155, 1986.

Brändström, U.: The Auroral Large Imaging System -Design, operation and scientific results. $\mathrm{PhD}$ thesis, Swedish Institute of Space Physics, Kiruna, Sweden, October 2003 (IRF Scientific Report 279), ISBN: 91-7305-405-4, 2003.

Carlson, C. W., Pfaff, R. F., and Watzin, J. G.: The Fast Auroral SnapshoT (FAST) mission, Geophys. Res. Lett., 25, 2013-2016, 1998.

Davis, T. N.: Observed characteristics of auroral forms, Space Sci. Rev., 22, 77-113, 1978.

Feldstein, Ya. I. and Galperin, Yu. I.: The auroral luminosity structure in the high-latitude upper atmosphere: its dynamics and relationship to the large-scale structure of the earths magnetosphere, Rev. Geophys., 23, 217-275, 1985.

Hedin, A. E.: Extension of the MSIS thermosphere model into the middle and lower atmosphere, J. Geophys. Res., 96, 1159-1172, 1991.

Horne, R. B. and Thorne, R. M.: Electron pitch angle diffusion by electrostatic electron cyclotron harmonic waves: The origin of pancake distributions., J. Geophys. Res., 105, 5391-5402, 2000.

Horne, R. B., Thorne, R. M., Meredith, N. P., and Anderson, R. R.: Diffuse auroral electron scattering by electron cyclotron harmonic and whistler mode waves during an isolated substorm., J. Geophys. Res., 108(A7), 1290, doi:10.1029/2002JA009736, 2003.

Ivanov, V. E., Kirillov, A. S., Sergienko, T. I., and Steen, Å.: Modelling of the altitude distribution of green line $(\lambda 5577 \AA)$ luminosity in aurora, in Airglow and Aurora, Sergej Leontyev, Editor., Proc. SPIE, 2050, 105-113, 1993.

Johnstone, A. D., Walton, D. M., Liu, R., and Hardy, D. A.: Pitch Angle Diffusion of Low-Energy Electrons by Whistler Mode Waves, J. Geophys. Res., 98, 5959-5967, 1993.

Kennel, C. F. and Petscheck, H. E.: Limit on stably trapped particle fluxes, J. Geophys. Res., 71, 1-28, 1966.

Kennel, C. F., Scarf, F. L., Fredricks, R. W., McGehee, J. H., and Coroniti, F. V.: VLF Electric Field Observations in the Magnetosphere, J. Geophys. Res., 75, 6136-6152, 1970.

Lui, A. T. Y. and Anger, C. D.: A uniform belt of diffuse auroral emission seen by the ISIS-2 scanning photometer, Planet. Space Sci., 21, 799-809, 1973.
Lyons, L. R., Koskinen, H. E. J., Blake, J. B., Egeland, A., Hirahara, M., Øieroset, M. Sandholt, P. E., and Shiokawa, K.: Processes leading to plasma losses into the high-latitude atmosphere, in: Magnetospheric Plasma Sources and Losses, edited by: Hultqvist, B., Øieroset, M., Pashmann, G., and Treumann, R., Vol. 6 of Space Science Series of ISSI, Dordrecht, Boston and London: Kluwer Academic Publishers, Chapt. 3, p. 85, 1999.

Lyons, L. R.: Pitch angle and energy diffusion coefficients from resonant interactions with ion-cyclotron and whistler waves, J. Plasma Phys., 12, 417-432, 1974.

Meredith, N. P., Horne, R. B., Johnstone, A. D., and Anderson, R. R.: The temporal evolution of electron distributions and associated wave activity following substorm injections in the inner magnetosphere, J. Geophys. Res., 105, 12 907-12 917, 2000.

Newell, P. Y., Feldstein, Y., Galperin, Y., and Meng, C.-I.: The morphology of nightside precipitation, J. Geophys. Res., 101, 10737-10 748, 1996.

Peticolas, L. M., Hallinan, T. J., Stenbaek-Nielsen, H. C., Bonnell, J. W., and Carlson, C. W.: A study of black aurora from aircraftbased optical observations and plasma measurements on FAST, J. Geophys. Res., 107(A8), 1217, doi:10.1029/2001JA900157, 2002.

Røyrvik, O.: Pulsating aurora: Local and global morphology, Ph.D. Thesis Alaska Univ., College., 1976.

Safargaleev, V. V. and Maltsev, Y. P.: Internal gravity waves in a plasma sheet, Geomagn. Aeronomy, 26, 270-274, 1986.

Sergienko T. I. and Ivanov, V. E.: A new approach to calculate the excitation of atmospheric gases by auroral electrons, Ann.Geophys., 11, 717-727, 1993.

Syrjäsuo, M. T.: FMI All-Sky Camera Network, Geophysical Publications, Finnish Meteorological Institute, ISBN 951-697-543-7, ISSN 0782-6087, 34 p., 2001.

Villalón, E. and Burke, W. J.: Pitch angle scattering of diffuse auroral electrons by whisler mode waves., J. Geophys. Res., 100, 19361-19369, 1995.

Vondrak, R. R., Murphree, J. S. Anger, C. D., and Wallis, D. D.: Ionospheric characteristics of a detached arc in the eveningsector trough., Geophys. Res. Lett, 10, 561-564, 1983.

Wallis, D. D., Burrows, J. R., Moshupi, M. C., Anger, C. D., and Murphree, J. S.: Observations of particles precipitating into detached arcs and patches equatorward of the auroral oval, J. Geophys. Res., 84, 1347-1360, 1979. 